\title{
Neue Therapiekonzepte mit Docetaxel in der neoadjuvanten (präoperativen) Behandlung des Mammakarzinoms
}

\author{
Docetaxel: New Therapeutic Concepts in the Neoadjuvant \\ (Preoperative)Treatment of Breast Cancer
}

\author{
M. Kaufmann \\ Klinik für Gynäkologie und Geburtshilfe, J ohann-Wolfgang-Goethe-Universität, Frankfurt/M.
}

\section{Rationale der präoperativen Chemotherapie}

Noch bis in die 60er Jahre wurde das Mammakarzinom als lokale Erkrankung aufgefasst. E rst in den 70er Jahren setzte sich die E rkenntnis durch, dass die M ehrzahl der M ammakarzinome Systemerkrankungen sind. Dies hatte nachhaltigen E influss auf die E ntwicklung der Therapiekonzepte. Vor allem wurde die Radikalität des operativen E ingriffs zugunsten systemischer Therapieansätze eingeschränkt. Die Rolle der primären (präoperativen) Chemotherapie wurde in den 80er Jahren ausschließlich darin gesehen, durch Reduzierung der $M$ asse sehr großer Tumoren die Operabilität zu verbessern bzw. die R ate brusterhaltender E ingriffe zu erhöhen. H eute kann durch die präoperative Chemotherapie die Chemosensitivität desTumors in vivo überprüft werden. A uf diese Weise erhofft man sich, die E ffektivität der adjuvanten Chemotherapie erhöhen zu können, denn deren Wirksamkeit lässt sich im postoperativen Z ustand nicht unmittelbar beurteilen. Außerdem soll die präoperative Chemotherapie dazu beitragen, die $\mathrm{K}$ rankheit systemisch zu bekämpfen, d.h. M ikrometastasen in einem sehr frühen $\mathrm{K}$ rankheitsstadium zu eliminieren, wenn die Tumorzellen noch keine R esistenzen entwickelt haben.

D ie Fragestellungen beim E insatz der präoperativen Chemotherapie im $\mathrm{R}$ ahmen klinischer Studien lassen sich unmittelbar aus den genannten Zielen ableiten. So gilt es zu klären, ob sich die $R$ ate brusterhaltender E ingriffe durch die B ehandlung erhöhen, das rezidivfreie Intervall und die Ü berlebenszeit verlängern und möglicherweise sogar die Heilungsrate steigern lassen. D arüber hinaus stellt sich die Frage, in welcher Weise die operative und lokoregionäre Vorgehensweise, aber auch postoperative (adjuvante) Maßnahmen die Effektivität des therapeutischen Gesamtkonzepts verbessern können. Des weiteren ist die Identifizierung neuer klinischer oder biologischer Prognosefaktoren beim Einsatz der neoadjuvanten Therapie eminent wichtig, verliert doch der bislang wichtigste Prognosefaktor, der operative Lymphknotenstatus, durch ein präoperatives D ownstaging erheblich an B edeutung.

\section{NSABP-Studien}

E ine der Schlüsselstudien zur neoadjuvanten Chemotherapie ist die randomisierte Phase-III-Studie B-18 des National Surgical A djuvant Breast and Bowel Project (NSA BP) [1]. Z wischen O ktober 1988 und A pril 1993 wurden 1523 Patientinnen mit operablem M ammakarzinom in die Studie aufgenommen. Sie wurden zwischen einem Standardarm mit O peration und vier adjuvanten Zyklen Doxorubicin/Cyclophosphamid (AC) und einem experimentellen A rm mit 4 präoperativen Zyklen AC und anschließender O peration randomisiert. Postmenopausale Patientinnen erhielten außerdem adjuvant Tamoxifen. Die Gesamtansprechrate auf die präoperative Chemotherapie betrug 79\% (9\% pathologisch komplette R emissionen), und eine brusterhaltende 0 peration war signifikant häufiger möglich als im Standardarm, vor allem bei den Patientinnen mit ursprünglich großen Tumoren von $\geq 5 \mathrm{~cm}$. A ußerdem führte die präoperative Chemotherapie bei $37 \%$ mehr Patientinnen zu einem pathologisch negativen Lymphknotenstatus. Wichtigstes Ergebnis war jedoch, dass nach einem 5jährigen Follow-up das rezidivfreie Ü berleben (jeweils $67 \%$ ) und das Gesamtüberleben (jeweils $80 \%$ ) in beiden Therapiearmen identisch war.

Den Ergebnissen dieser Studie zufolge ist die präoperative Chemotherapie beim primär operablen M ammakarzinom eine genauso sichere und wirksame B ehandlungsform wie die adjuvante Therapie. Sie induziert in vielen Fällen einen nodalnegativen Lymphknotenstatus und ermöglicht es, häufiger brusterhaltend zu operieren. E ine primäre Tumorprogression ist eine seltene Ausnahme. A ndererseits ist das komplette A nsprechen auf die Chemotherapie mit einer besseren Ü berlebensprognose korreliert.

Konsequenterweise wird in der neoadjuvanten Nachfolgestudie N SA B P B -27 in allen drei Therapiearmen eine präoperative $C$ hemotherapie eingesetzt ( 4 Z yklen $A C$ zusammen mit dem Beginn einer Hormontherapie mit Tamoxifen $20 \mathrm{mg}$ für die Dauer von 5 Jahren). Im ersten Studienarm ist das die

\begin{tabular}{ll}
\hline KARGER & $\odot$ 2000 S. K arger G mbH , Freiburg \\
$\begin{array}{l}\text { Fax +49 761 4520714 } \\
\begin{array}{l}\text { E-mail Information@K arger.de } \\
\text { www.karger.com }\end{array}\end{array}$ & A ccessible online at: \\
www.karger.com/journals/onk
\end{tabular}

Prof. Dr. M anfred K aufmann

Klinik für G ynäkologie und G eburtshilfe

J ohann-Wolfgang-G oethe-U niversität

Theodor-Stern-K ai 7

D -60596 Frankfurt/M . (G ermany) 
einzige präoperative Therapie, während im zweiten A rm im A nschluss an AC noch 4 präoperative Zyklen D ocetaxel verabreicht werden. Im dritten Studienarm erhalten die Patientinnen 4 Zyklen D ocetaxel postoperativ (adjuvant).

$\mathrm{U} \mathrm{m}$ die $\mathrm{E}$ rgebnisse der präoperativen Therapie zu optimieren, gilt es in erster $L$ inie die $R$ ate pathologisch kompletter R emissionen zu erhöhen. Dies erscheint auf verschiedene Arten möglich:

- Kombination bestmöglich aufeinander abgestimmter Tumorwirkstoffe, wobei nach den Erfahrungen in der Therapie des metastasierten Mammakarzinoms Taxane und A nthrazykline eine wesentliche R olle spielen werden;

- D osisintensivierung oder Verlängerung der Therapie;

- individuell ausgerichteteTherapieansätze bei primär resistenten Tumoren.

\section{Aberdeen-Studie}

$D$ as Problem der Tumorresistenz fand in einer Studie der $A$ berdeen $B$ reast $G$ roup von $E$ remin und $M$ itarbeitern besondere Berücksichtigung. In diese Studie wurden 163 Patientinnen mit großen Primärtumoren $(>3 \mathrm{~cm}$ ) oder lokal fortgeschrittener Erkrankung $\left(\mathrm{T}_{3}, \mathrm{~T}_{4}, \mathrm{~T}_{\mathrm{x}} \mathrm{N}_{2}\right)$ aufgenommen. A lle erhielten in einer ersten Phase präoperativ zunächst 4 Zyklen CVA P (Cyclophosphamid, V incristin, D oxorubicin, PrednisoIon). Patientinnen, die auf diese Therapie ansprachen, wurden in der zweiten Studienphase randomisiert: E ntweder weitere 4 präoperative Zyklen CVA P oder stattdessen 4 Zyklen D ocetaxel. Patientinnen, die in der ersten Studienphase durch CVA $P$ nicht in eine R emission gelangten, erhielten alle ohne $\mathrm{R}$ andomisierung in der zweiten Phase 4 präoperative Zyklen D ocetaxel. E rste E rgebnisse dieser Studie wurden auf dem B reast Cancer Symposium 1999 in San A ntonio vorgestellt. A uf die ersten 4 präoperativen Zyklen CVA P sprachen $65 \%$ der Patientinnen an, $14 \%$ mit einer kompletten Remission. Von den 49 Non-R espondern sprachen immerhin noch $47 \%$ auf D ocetaxel an, $10 \%$ erreichten sogar eine komplette R emission. In den beiden randomisierten Studienarmen ( $d$.h. bei den primären CVA P-R espondern) ergab sich folgende Situation: M it 4 weiteren Zyklen CVAP wurde schließlich bei $33 \%$ der Patientinnen eine Komplettremission erreicht (G esamtansprechrate $67 \%$ ), mit D ocetaxel jedoch bei $61 \%$ der Patientinnen (G esamtansprechrate 94\%). Dieser U nterschied war signifikant $(p=0,001)[2]$.

\section{GEPARDO und GEPAR-DUO}

In Deutschland initiierte die GABG V (German A djuvant Breast Cancer Group) die randomisierte Phase-II-Studie GEPA R D O, deren R ekrutierungsphase im J uni 1999 nach nur 14 Monaten abgeschlossen werden konnte. Die insgesamt 250 Patientinnen $\left(T \geq_{3} \mathrm{~cm} N_{0-2} M_{0}\right.$ ) wurden zwischen zwei Studienarmen randomisiert: 4 präoperative dosisintensivierte Zyklen A D oc (D oxorubicin $50 \mathrm{mg} / \mathrm{m}^{2}+$ D ocetaxel $75 \mathrm{mg} / \mathrm{m}^{2}$ alle 2 Wochen, G-CSF an den Tagen $6-10$ jedes Zyklus) mit oder ohne präoperatives Tamoxifen ( $30 \mathrm{mg} / \mathrm{m}^{2}$ p.o. $1 \times$ täglich ab dem ersten Tag der Chemotherapie). Nach der O peration
Tab.1. A nsprechen des Primärtumors auf die präoperative Chemotherapie mit 4 Zyklen D oxorubicin und D ocetaxel (A D oc) mit oder ohne Tamoxifen. E rgebnisse der GE PA R D O-Studie (mod. nach K aufmann)

\begin{tabular}{llrlll}
\hline M ethode & \multicolumn{2}{c}{ Response, \% } & & & \\
& $n$ & CR & PR & SD & PD \\
\hline Palpation & 247 & 27,9 & 52,9 & 16,3 & 2.9 \\
H istologie & 240 & 7,9 & 66,3 & 23,0 & 2.8 \\
U Itraschall & 178 & 9,7 & - & - & -
\end{tabular}

$\mathrm{CR}=$ Complete response, $\mathrm{PR}=$ partial response, $\mathrm{SD}=$ stable disease, $\mathrm{PD}=$ progressive disease.

(und einer Strahlentherapie bei brusterhaltend operierten Chemotherapie-R espondern) erhalten alle Patientinnen für 5 Jahre Tamoxifen. D as 2wöchige A D oc-Schema war zuvor in einer Pilotstudie auf seine Praktikabilität hin geprüft worden und hatte sich als gut verträglich und hochwirksam erwiesen [3] (Remissionsrate $93 \%$ bei palpatorischer bzw. $67 \%$ bei sonographischer A uswertung der Tumorgröße). Dies wurde durch die G E PA R D O -D aten bestätigt. D ie palpatorisch beurteilte Gesamtansprechrate betrug $81 \%$ mit $28 \%$ kompletten R emissionen. D ie sonographisch bzw. histologisch bestimmte CR - R ate lag bei 8 bzw. 10\% [4] (Tab. 1).

D iesen Ergebnissen zufolge ist die Kombination ADoc ein mäßig toxisches, praktikables und hochwirksames Chemotherapieregime für die neoadjuvante Therapie des primär operablen M ammakarzinoms. D urch die schnelle Zyklusfolge können die Patientinnen bereits 9-10 Wochen nach Beginn der Chemotherapie operiert werden. D ie Verträglichkeit wird durch die zusätzliche Verabreichung von Tamoxifen nicht beeinflusst. D ie B eurteilung der W irksamkeit (rezidivfreies und G esamtüberleben) in A bhängigkeit vom Therapiearm steht noch aus. A us den E rfahrungen dieser Studie wurde aber bereits das Konzept der neuen Phasen-III-Studie G E PA R -D U O entwickelt. D ie R ekrutierung für diese Studie, die insgesamt 1000 Patientinnen mit einer Tumorgröße von $\geq 2 \mathrm{~cm}$ umfassen soll, wurde im Juni 1999 begonnen. Bislang wurden etwa 300 Patientinnen aufgenommen. Die Behandlung im ersten Therapiearm entspricht der der GE PA RD 0-Studie ( 4 Zyklen $A$ D oc alle 2 Wochen), die im zweiten $A$ rm dem sequenziellen Protokoll der Studie NSA BP B-27 mit 4 Zyklen A C (D oxorubicin $50 \mathrm{mg} / \mathrm{m}^{2}$ + Cyclophosphamid $600 \mathrm{mg} / \mathrm{m}^{2}$ ) alle 3 Wochen, gefolgt von 4 Zyklen Docetaxel $100 \mathrm{mg} / \mathrm{m}^{2}$ alle 3 Wochen. A lle Patientinnen erhalten ab dem ersten Tag der Chemotherapie Tamoxifen.

\section{Schlussfolgerungen}

D ie präoperative Chemotherapie ist nach wie vor eine experimentelle Behandlungsform, die jedoch auf einem soliden theoretischen Fundament steht und durch die bislang vorliegenden klinischen E rgebnisse gestützt wird. Für die Verbesserung der neoadjuvanten Therapie und die Identifizierung prädiktiver R esponsemarker sind jedoch weitere großangelegte randomisierte Studien erforderlich. Für deren rasche Durch- 
führung ist die M itarbeit möglichst vieler Ä rzte und die zügige Rekrutierung von Patientinnen unumgänglich. Docetaxel ist derzeit eines der wirksamsten Zytostatika beim metastasierten Mammakarzinom, so dass auch auf seiner Integration in präoperative Therapieregime bei Patientinnen mit großen Primärtumoren bzw. lokal fortgeschrittener E rkrankung große $\mathrm{H}$ offnungen ruhen.

\section{Literatur}

\begin{abstract}
1 Fisher $B, B$ rown $A, M$ amounas $E$, Wieand $S, R$ obidoux $A$, M argolese R G, Cruz A B J r, Fisher E R, Wickerham $D L$, Wolmark N, DeCillis A, Hoehn JL, Lees AW, Dimitrov NV: Effect of preoperative chemotherapy on local-regional disease in women with operable breast cancer: findings from $\mathrm{N}$ ational Surgical A djuvant Breast and Bowel Project B-18. J Clin Oncol 1997:15:2483-2493.
\end{abstract}

2 H utcheon A, O gston K, H eys S, Smith I, Whitaker T, M iller I, Payne S, A h-See A, E remin O, E ggleton P: Primary chemotherapy in the treatment of breast cancer: Significantly enhanced clinical and pathological response with docetaxel. Proc A m Soc Clin O ncol 2000:317.

3 von M inckwitz G, Costa SD, E iermann W, Blohme $\mathrm{JU}$, Tulusan $\mathrm{A} H$, Jackisch $\mathrm{C}, \mathrm{K}$ aufmann $\mathrm{M}: \mathrm{M}$ aximized reduction of primary breast tumor size using preoperative chemotherapy with doxorubicin and docetaxel. J Clin O ncol 1999;17:1999-2005.

4 von Minckwitz G, Costa SD, Raab G, et al: Randomized trial on preoperative dose-intensified A driamycin-docetaxel (A D oc) vs. A D oc + tamoxifen in primary operable breast cancer. 22nd A nnual San A ntonio B reast Cancer Symposium, 8.-11.12.1999, Poster 247. 